\title{
A New Polystyrene-based
}

\section{Ionomer/MWCNT Nanocomposite for}

\section{Wearable Skin Temperature Sensors}

Alessio Giuliani, ${ }^{1}$ Massimo Placidi, ${ }^{2}$ Fabio Di Francesco, ${ }^{1,3}$ Andrea Pucci, ${ }^{1,3}$

${ }^{1}$ Dipartimento di Chimica e Chimica Industriale, Università di Pisa, Via Risorgimento 35, 56126 Pisa, Italy;

${ }^{2}$ HORIBA Jobin Yvon S.r.1., Via Cesare Pavese 19/21 - 20090 Opera, Milano, Italy

${ }^{3}$ INSTM, Unità di Ricerca di Pisa, Via Risorgimento 35, 56126 Pisa, Italy

Corresponding author:

Dr. Andrea Pucci

Dipartimento di Chimica e Chimica Industriale, Università di Pisa, Via Risorgimento 35, 56126 Pisa, Italy. Tel: +39 050 2219270; Fax: +39 0502219 260; Email: apucci@dcci.unipi.it 


\begin{abstract}
The present work outlines the fabrication and testing of a novel skin temperature sensor based on exfoliated and undamaged multi-walled carbon nanotubes (MWCNTs) dispersed in a poly(vinylbenzyl chloride) derivative with triethylamine $\left(\mathrm{PVBC}_{-} \mathrm{Et}_{3} \mathrm{~N}\right)$. The dispersions were prepared by sonicating MWCNT/PVBC_ $\mathrm{Et}_{3} \mathrm{~N}$ mixtures in dimethylformamide for 5 min and the quantification of the MWCNTs dispersed was evaluated by UV-vis spectroscopy investigations and thermogravimetric analyses.

The investigations demonstrated the realization of $\mathrm{MWCNT} / \mathrm{PVBC} \mathrm{Et}_{3} \mathrm{~N}$ sensors with a resistance sensitivity to temperature close to $-0.004 \mathrm{~K}^{-1}$, an absolute value that is comparable to the highest values found in metals. The temperature dependence of the resistance was also found very reproducible in the range $20-40{ }^{\circ} \mathrm{C}$, thus suggesting the possibility of using the MWCNT/PVBC_Et ${ }_{3} \mathrm{~N}$ system as for the fabrication of small wearable temperature sensors to be used for the monitoring of chronic wounds.
\end{abstract}

Keywords: MWCNTs, poly(vinylbenzyl chloride) ionomers, MWCNT dispersion, electrically conducting nanocomposite, wearable temperature sensor 


\section{Introduction}

Carbon nanotubes (CNTs) are a third allotropic form of carbon brought to the forefront by the pioneering work of Iijima et al. in 1991.[1] CNTs represent a dominant class of nanostructured materials whose unique mechanical, electrical and thermal properties depend critically on their structural perfection and high aspect ratio (typically $>10^{2}$ ). [2-4] Single-walled CNTs (SWCNTs) consist of single graphene sheets (monolayer of $\mathrm{sp}^{2}$ bonded carbon atoms) wrapped into cylindrical tubes with a diameter ranging from 0.7 to $2 \mathrm{~nm}$ and lengths up to micrometers. Multi-walled CNTs (MWCNTs) consist of concentric assemblies of SWCNTs and are therefore characterized by larger average diameters. The exceptional properties shown by SWCNTs and MWCNTs support the virtues of their incorporation into polymeric matrices to produce nanocomposites for a variety of applications, ranging from strong and lightweight materials to nanometer-sized electronic devices and electrochemical sensors.[2, 5-9]

However, even if CNTs potentially represent one of the most important filler materials for polymers, their utilization is problematic due to the strong van der Waals interactions between individual nanotubes, which hinder a uniform dispersion at the nanoscale level. Ultrasonication of CNTs mixtures containing the desired polymer is mostly used for unbundling CNTs, even if significant damage of their structures as well as shortening occur, which limit the full potential of CNTs as additives in polymers.[10]

We have recently investigated the influence of the sonication time on the characteristics of MWCNTs dispersions within poly(styrene-b-(ethylene-co-butylene)-b-styrene) (SEBS) mixtures for the realization of miniaturized temperature sensors.[11, 12] We demonstrated that a sonication time higher than 30 min induced an extensive MWCNTs degradation (average length decreased of about $40 \%$, which affected the electrical conductivity of the nanocomposites. On the other hand, the use of alkyl-functionalized MWCNTs appeared to be more effective in preparing SEBS nanocomposites due to the higher dispersion efficiency, negligible nanotube degradation and higher electrical conductivity. The resulting films showed a temperature dependent resistivity with a 
sensitivity comparable to the highest values found in metals, which was however partly lost after the first heating cycle up to $55-60{ }^{\circ} \mathrm{C}$.[11] This loss of sensitivity was attributed to the elastomeric nature of the SEBS matrix, whose mobility with temperature did not ensure a phase stability to the dispersed MWCNTs.

In this work, aiming at preparing highly dispersed MWCNT/polymer nanocomposites with a more stable temperature sensitivity in multiple cycles, we selected a polystyrene-based ionomer (a poly(vinylbenzyl chloride) (PVBC) aminated with triethylamine, $\mathrm{PVBC}_{-} \mathrm{Et}_{3} \mathrm{~N}$ ) as the continuous polymer matrix. Indeed, the use of surfactants and ionic polymers (ionomers) is well reported in literature for the realization of high concentration, high-quality CNT dispersions for technological applications.[13-15] The ionomers are reported to effectively interact with CNTs through weak van der Waals and cation- $\pi$ interactions. Once the CNTs are detached during sonication, they are immediately surrounded by the ionic macromolecules thus shielding the strong $\pi-\pi$ interactions between CNTs and eventually preventing their rebundling.[16, 17]

PVBC is an ideal, high glass transition temperature $(\mathrm{Tg})$ polymer $\left(\sim 100{ }^{\circ} \mathrm{C}\right)$ to which a variety of functional moieties can be attached by nucleophilic substitutions in mild reaction conditions.[18-21] Moreover, the high $\mathrm{Tg}$ of these materials should guarantee the phase stability of CNT nanocomposites in the low temperature regime $\left(20-40{ }^{\circ} \mathrm{C}\right)$, as the application target of the present research is the realization of wearable temperature sensors for the monitoring of chronic wounds.

The dispersions were prepared by sonicating MWCNT/PVBC_Et $3 \mathrm{~N}$ mixtures in DMF for 5 min, and the amount of dispersed CNTs was quantified by UV-vis absorbance measurements and gravimetric analyses. Atomic force microscopy and Raman spectroscopy were employed to assess the structural integrity of MWCNTs. The temperature dependence of resistance of MWCNT/PVBC_Et 3 N nanocomposites was investigated in the range $20-60{ }^{\circ} \mathrm{C}$ to explore their potential for the development of linear, stable and reproducible temperature sensors. 


\section{Materials and methods.}

\subsection{Materials}

All the solvents were either used as received or purified by standard procedures.

4-Vinylbenzyl chloride (VBC) (4-(chloromethyl)styrene, Aldrich, 90\%) was purified by washing it twice with a $25 \%(\mathrm{v} / \mathrm{v})$ aqueous sodium hydroxide solution, and then thoroughly washed with distilled water until neutral. Finally, the monomer was distilled at reduced pressure and stored under nitrogen over molecular sieves at $-20{ }^{\circ} \mathrm{C} . \alpha, \alpha^{\prime}$-Azobisisobutyronitrile (AIBN) (Carlo Erba) was purified by recrystallization from acetone. Triethylamine (TMA) (Sigma-Aldrich, $\geq 99 \%$ ) was used as received.

Multi-walled carbon nanotubes (MWCNTs, Baytubes C150 P), a generous gift from Bayer Material Science, were used as received. These nanotubes are vapor grown and typically consist of 3-15 graphitic layers wrapped around a hollow $4 \mathrm{~nm}$ core. Typical diameters range from 13 to $16 \mathrm{~nm}$, and the lengths are between 1 and $10 \mu \mathrm{m}$. The density is approximately $1.4-1.6 \mathrm{~g} / \mathrm{cm}^{3}$.

\subsection{Preparation of $P V B C$}

Poly(vinylbenzyl chloride) (PVBC) was prepared by radical bulk polymerization of VBC in the presence of $1 \%$ by mol of AIBN. The solution was stirred at $70{ }^{\circ} \mathrm{C}$ for two hours under nitrogen atmosphere, the crude product was then purified by a double dissolution in chloroform and precipitation in methanol and recovered after drying at reduced pressure. The monomer conversion $(\sim 30 \%)$ was determined off-line via gravimetric analysis and the molecular weight of the sample was evaluated by size exclusion chromatography.

\subsection{Preparation of $\mathrm{PVBC} \mathrm{Et}_{3} \mathrm{~N}$}

The purified PVBC $(0.543 \mathrm{~g}, 3.56 \mathrm{mmol}$ of VBC repeating units $)$ was dissolved in $20 \mathrm{~mL}$ of chloroform in the presence of a two-fold molar excess of $\mathrm{Et}_{3} \mathrm{~N}(1 \mathrm{~mL}, 0.720 \mathrm{~g}, 0.712 \mathrm{mmol})$ and the solution stirred at $60{ }^{\circ} \mathrm{C}$ for 24 hours during which the solution became turbid. The reaction mixture was concentrated by evaporating chloroform, and $0.910 \mathrm{~g}$ of PVBC_Et ${ }_{3} \mathrm{~N}$ were finally obtained by precipitating the suspension twice in diethyl ether and drying the precipitate under vacuum. 


\subsection{Preparation and characterization of the MWNT/ $\mathrm{PVBC} \mathrm{Et}_{3} \mathrm{~N}$ dispersions}

A stock solution was prepared by dissolving PVBC_Et ${ }_{3} \mathrm{~N}(0.4 \mathrm{~g})$ in $100 \mathrm{~mL}$ of dimethylformamide $(100 \mathrm{~mL})$. Aliquots of this solution $(5 \mathrm{~mL})$ were poured in vials containing different amounts of MWCNTs, up to a 1:1 weight ratio with PVBC_Et ${ }_{3} N$. Uniform dispersions were then obtained by ultrasonicating (UP $400 \mathrm{~S}$, Hielscher) the mixtures for $5 \mathrm{~min}$ at full power $(400 \mathrm{~W})$ and at a frequency of $24 \mathrm{kHz}$. The immersion of the vials in a water/ice bath allowed us to keep the evaporation of toluene during sonication at negligible levels. The resulting dispersions were centrifuged at $4000 \mathrm{rpm}$ for $30 \mathrm{~min}$ and then filtered to remove the residual MWCNT agglomerates. The final MWCNTs/ PVBC_Et ${ }_{3} \mathrm{~N}$ weight ratio in the mixture was evaluated by thermogravimetric analysis (TGA) on the dried materials. The MWCNTs wt.\% was determined by calculating the ratio between the degradation steps of PVBC_Et $3 \mathrm{~N}$ (from room temperature to $600{ }^{\circ} \mathrm{C}$ ) and $\mathrm{MWCNT}$ (from $600{ }^{\circ} \mathrm{C}$ to $900{ }^{\circ} \mathrm{C}$ ).

The electric resistance measurements were performed on films obtained by casting $10 \mu \mathrm{L}$ of the MWCNT/PVBC_Et ${ }_{3} \mathrm{~N}$ dispersions in DMF onto a gold electrode pair supported on a $50 \mu \mathrm{m}$ thick polyimide film (Cad Line, Pisa, Italy). Polyimide was chosen due to flexibility and chemical inertness, which are important constraints for wearable temperature sensors to be used for the monitoring of chronic wounds. Gold electrodes were prepared from copper tracks obtained by photolithography, which were subsequently electroplated with nickel and gold (electrode dimensions: length $7 \mathrm{~mm}$, width $1 \mathrm{~mm}$, distance $2 \mathrm{~mm}$; thickness of copper $35 \mu \mathrm{m}$, nickel $3.0 \mu \mathrm{m}$, gold $1.2 \mu \mathrm{m})$.

\subsection{Apparatuses}

Infrared spectra were recorded by a Fourier transform infrared spectrometer (Spectrum One, PerkinElmer) on films cast from $\mathrm{CHCl}_{3}$ or $\mathrm{DMF}$ solutions on a $\mathrm{KBr}$ window.

Proton magnetic resonance $\left({ }^{1} \mathrm{H}\right.$ NMR) spectra were recorded by using a Varian Gemini $300 \mathrm{MHz}$ on $5-10 \% \mathrm{CDCl}_{3}$ (Aldrich, 99.8 atom \% D) solutions. NMR spectra were registered at $20{ }^{\circ} \mathrm{C}$ and the chemical shifts were assigned in ppm using the solvent signal as a reference. Data analysis was 
performed by in-house routines developed on the Igor Pro platform Wavemetrics. Curve fitting was done by least-squares methods using built-in functions in Igor Pro $\left(\mathrm{R}^{2}=0.998\right)$.

The PVBC molecular weight was obtained with high performance liquid chromatography analyses of chloroform-diluted solutions performed by an isocratic pump unit (PU-1580, Jasco), a refractive index detector (830-RI, Jasco) and two organic GPC/SEC columns (PLgel $5 \mu \mathrm{m}$ MIXED- C, Polymer Laboratories). The calibration curve was obtained with polystyrene standard samples.

The morphologies of MWNTs/PVBC_Et $3 \mathrm{~N}$ films were examined by a multimode atomic force microscope (AFM, Veeco Metrology, Inc.) equipped with E and J piezoelectric scanners. The instrument was connected to controller and to a signal direct access module (Veeco Metrology Inc. Nanoscope IV) connected to a oscilloscope (TDS 2004B Tektronix).

The ultraviolet-visible (UV-vis) absorption spectra of dispersions were recorded with a UV/vis spectrometer (Perkin-Elmer Lambda 650).

Thermogravimetric scans were carried out by a Mettler Toledo Starc System (TGA/SDTA851 ${ }^{\mathrm{e}}$ ). Samples were heated from 25 to $600{ }^{\circ} \mathrm{C}$ at $10{ }^{\circ} \mathrm{C} / \mathrm{min}$ under a nitrogen flow, and from 600 to 900 ${ }^{\circ} \mathrm{C}$ under an air flow.

Raman spectroscopy was performed using a confocal Raman microscope (Horiba Jobin Yvon Xplora ONE). The wavelength of the excitation laser was $785 \mathrm{~nm}$ and the power of the laser was kept below $1 \mathrm{~mW}$ to avoid any noticeable sample heating. The laser spot size was $1 \mu \mathrm{m}$ with a $100 \mathrm{X}$ objective lens (numerical aperture D 0:90). The signal was acquired using a thermoelectrical CCD cooled down to $-50{ }^{\circ} \mathrm{C}$.

Calorimetric analyses were performed under nitrogen flux $(80 \mathrm{~mL} / \mathrm{min})$ by a differential scanning calorimeter (Mettler-Toledo 822e) equipped with a cooling system. The calibration was performed with Zinc and Indium. Heating and cooling thermograms were recorded at a standard rate of $10^{\circ} \mathrm{C} / \mathrm{min}$

The sensitivity to temperature of nanocomposites was evaluated by sandwiching the films between two aluminium plates heated by two mica plate heaters (RS 615-1700, RS Components). A 
temperature controller (dTRON 316, Jumo) connected a thermocouple embedded in one of the plates allowed a fine regulation of the set-point temperature. Film resistance was measured by a precision digital multimeter (2700, Keithley).

\section{Results and discussion}

\subsection{Preparation and characterization of $P V B C \_E t_{3} N$}

Poly(vinylbenzyl chloride) (PVBC) was prepared by radical bulk polymerization of VBC in the presence of $1 \%$ by mol of AIBN under nitrogen atmosphere. The polymer was characterized by a number- and weight-average molecular weight of 12,000 $\mathrm{g} \mathrm{mol}^{-1}$ and 20,400 $\mathrm{g} \mathrm{mol}^{-1}$ respectively, and a monomer conversion of about $30 \%$. The glass transition temperature was about $100{ }^{\circ} \mathrm{C}$.

PVBC was then converted in the aminated PVBC_Et ${ }_{3} \mathrm{~N}$ form with a two-fold molar excess of triethylamine. The FT-IR spectra in Figure 1 reveal a significant decrease of the $-\mathrm{CH}_{2} \mathrm{Cl}$ and C-Cl stretching bands at $1265 \mathrm{~cm}^{-1}$ and $675 \mathrm{~cm}^{-1}$ and the emersion of the $\mathrm{C}-\mathrm{N}$ peak at $1370 \mathrm{~cm}^{-1}$ after the reaction of $\mathrm{PVBC}$ with $\mathrm{Et}_{3} \mathrm{~N}$.

$<$ Figure 1 near here $>$

The intense band near $1600 \mathrm{~cm}^{-1}$ was attributed to the bending related to absorbed water and indicates the hydrophilic character of the PVBC_Et ${ }_{3} \mathrm{~N}$ system.

The ${ }^{1} \mathrm{H}$ NMR spectrum of PVBC (Figure 2a) displays peaks in the range 7.1-6.5 ppm and 2-0.9 ppm that were referred to the aromatic and aliphatic protons of the VBC repeating units, and a peak at about $4.5 \mathrm{ppm}$ that was attributed to the two chloromethylenic protons of VBC. After reaction with $\mathrm{Et}_{3} \mathrm{~N}$, a new broad resonance emerged at $4.4 \mathrm{ppm}$ due to the ammonium nitrogen-linked methylene protons $-\mathrm{CH}_{2}-\mathrm{N}^{+} \mathrm{Et}_{3}$ (Figure 2b). This broad and asymmetric peak was caused by a residual contribution at $4.5 \mathrm{ppm}$ due to the unreacted VBC units. Based on peak integrals calculated after a deconvolution analysis (dotted lines, Figure $2 \mathrm{~b}$ ), about $78 \% \mathrm{Cl}$ atoms of the benzyl groups in PVBC were replaced by $\mathrm{Et}_{3} \mathrm{~N}$.

$<$ Figure 2 near here $>$ 
After reaction with $\mathrm{Et}_{3} \mathrm{~N}$, the polymer exhibited a $\mathrm{Tg}$ of about $125^{\circ} \mathrm{C}$.

\subsection{Preparation and characterization of $M W C N T / P V B C \_E t_{3} N$ mixtures}

MWCNT/PVBC_Et ${ }_{3} \mathrm{~N}$ dispersions were obtained by ultrasonication of precursors in DMF, followed by centrifugation. The ultrasonic device was fixed so that its sonotrode could be reproducibly dipped into the dispersion, which was contained every time in vials of the same volume and shape. An effective refrigeration with a stirred water/ice bath limited the increase in temperature and the loss of solvent to negligible levels.

All CNTs are active in the UV-vis region and exhibit characteristic bands corresponding to 1D van Hove singularities.[22, 23] Therefore, it is possible to establish a relationship between the amount of CNTs individually dispersed in the PVBC_Et ${ }_{3} \mathrm{~N}$ DMF mixture and the intensity of the corresponding absorption spectrum. Figure 3 shows the UV-vis absorbance measurements of MWCNT/PVBC_Et 3 N DMF dispersions plotted at different MWCNT concentrations obtained after 5 min of sonication. The time of sonication was limited to 5 min to prevent CNTs degradation.[12] In literature, the absorbance spectrum of CNTs reveals intense absorption peaks at 255 and $273 \mathrm{~nm}$ attributed to bulk $\pi$-plasmon and surface excitations, respectively.[24] As a result of the strong overlapping spectral features of PVBC_Et $3 \mathrm{~N}$ and DMF, the cut-off was necessarily positioned at $320 \mathrm{~nm}$. The absorbance spectrum of pure PVBC_Et ${ }_{3} \mathrm{~N}$ in DMF solution does not display any significant absorption over the entire wavelength region of 320-500 $\mathrm{nm}$.

During sonication, the dissipated mechanical energy overcomes the van der Waals interactions in the MWCNTs bundles and leads to their debundling and dispersion. The increasing amount of MWCNT concentration resulted in an increasing value of the absorbance as shown in Figure 3, which gradually decreased from UV to near-IR. This decrease is also partly the result of scattering, especially in the lower wavelength range.

$<$ Figure 3 near here $>$ 
Thermogravimetric analyses permitted to quantify the amount of MWNTs included in the PVBC_Et ${ }_{3} \mathrm{~N}$ matrix. A typical result of a thermogravimetric scan (Figure S1, suppl. info) showed polymer degradation at about $210{ }^{\circ} \mathrm{C}$, with the loss of the ionomeric moieties, and at $425{ }^{\circ} \mathrm{C}$, whereas the burning of the residual carbon nanotubes is activated at $600{ }^{\circ} \mathrm{C}$ when the purge gas is switched from nitrogen to air.

In Figure 4a, the weight percentage of MWNTs in the PVBC_Et ${ }_{3} \mathrm{~N}$ nanocomposite is reported as a function of the MWNT concentration. Measurements performed in triplicate showed only a $2 \%$ relative standard deviation. A 13 wt.\% dispersion of MWNTs in the PVBC_Et ${ }_{3} \mathrm{~N}$ matrix was achieved for the MWCNT/PVBC_Et ${ }_{3} \mathrm{~N}$ 1:20 mixture. This amount suggests the excellent surfactant properties of the styrene-based ionomer, since equivalent MWCNT contents were previously obtained only with equiponderal mixtures of CNT and poly(styrene-b-(ethylene-co-butylene)-bstyrene) after 10 min of sonication.[12] This amount increased monotonically according to the UVvis results shown in Figure 3, up to about 47.5 wt.\% for the MWCNT/PVBC_Et ${ }_{3} \mathrm{~N}$ 1:1 experiment.

$<$ Figure 4 near here $>$

It is worth noting that plotting the absorbance at the given wavelength of $400 \mathrm{~nm}$ against the w/w percentage concentration of MWCNTs (data from TGA, Figure 4a) demonstrates a linear correlation $\left(\mathrm{R}^{2}=0.96\right)$, especially in the low concentration regime (Figure $\left.4 \mathrm{~b}\right)$. A deviation from linearity at higher MWCNT concentration could be attributed to the stronger contribution of scattering phenomena. The slope of the fitting line, i.e. the apparent absorption coefficient, was calculated to be $0.018 \pm 0.002 \mathrm{~mL}^{-1}$.

In order to evaluate if the dispersion process allowed the exfoliation of MWNTs, the MWCNT/PVBC_Et3N mixtures were analyzed by atomic force microscopy (AFM). Figure 5 shows a representative AFM image of the MWCNT/PVBC_Et3N 1:4 sample (19.3 wt.\% of MWCNT) deposited on mica. The image clearly indicates that most of CNTs are exfoliated into small bundles less than $15 \mathrm{~nm}$ in diameter or even individual nanotubes. This result suggests the main role of the ionomer in promoting favorable interactions with MWCNTs, thus favoring their 
debundling.

$<$ Figure 5 near here $>$

Raman spectroscopy is a versatile technique to study carbon nanotube composition and possible degradation during processing. $[25,26]$ The most prominent features in a Raman spectrum of MWCNTs are the G and D bands (Figure 6). The G-band appeared near $1600 \mathrm{~cm}^{-1}$ and corresponds to planar vibration of carbon atoms of most graphite like materials. G-band is thus an intrinsic feature of CNTs, closely related to vibrations in all $\mathrm{sp}_{2}$ carbon materials. The disorder-induced Dband appeared near $1310 \mathrm{~cm}^{-1}$ and was attributed to the scattering from defects, which break the basic symmetry of the graphene sheet. MWCNTs typically show the highest $\mathrm{I}_{\mathrm{D}} / \mathrm{I}_{\mathrm{G}}$ ratio, since large amounts of structural defects are present due to its multiple graphite layers. In our case, a $\mathrm{I}_{\mathrm{D}} / \mathrm{I}_{\mathrm{G}}$ ratio of 1.9 was calculated for the MWCNT/PVBC_Et ${ }_{3} \mathrm{~N}$ prepared both from 1:1 and 1:4 by weight mixtures in DMF, that was totally in agreement with the value of 2 measured for pristine MWCNTs.[27] This result indicates that the experimental set-up utilized for the preparation of MWCNT/PVBC_Et ${ }_{3} \mathrm{~N}$ nanocomposites did not affect the graphitic order on the MWCNTs surface, and further confirm that the limitation of the sonication time to 5 min preserves CNT structure.[12] Indeed, the so-called $\mathrm{G}^{\prime}$ band $\left(\sim 2610 \mathrm{~cm}^{-1}\right)$, associated with second-order $\mathrm{sp}_{2}$ carbon feature and reflecting the metallic character in MWCNTs,[28] is well detectable in the spectrum.

$<$ Figure 6 near here $>$

The electrical resistance of the composite was found to decrease according to the amount of MWNTs dispersed in the PVBC_Et ${ }_{3} \mathrm{~N}$ matrix (Figure 7).

$<$ Figure 7 near here $>$

A strong variation of resistance, i.e. from $94.5 \mathrm{k} \Omega$ to $2.6 \mathrm{k} \Omega$, occurred for a relative small variation of MWCNT concentration, i.e. from 13.2 wt. $\%$ to $17.6 \mathrm{wt} . \%$. After that, the resistance of the nanocomposite changed less markedly but still steadily, suggesting that the additional amount of dispersed nanotubes was still able to create new effective percolation pathways within the polymer matrix. 


\subsection{Resistance sensitivity to temperature}

CNTs with either semi-conducting or metallic character show a resistivity that depends on temperature, which makes CNT nanocomposites potentially useful for the fabrication of temperature sensors.

$<$ Figure 8 near here $>$

Figure 9 shows the calibration curves of sensors fabricated with MWCNT/PVBC_Et ${ }_{3} \mathrm{~N}$ dispersions containing different amounts of MWCNTs. A linear correlation of the resistance variation can be observed for all the prepared MWCNT/PVBC_Et ${ }_{3} \mathrm{~N}$ nanocomposites. More specifically, the sensors fabricated with the lowest MWCNT concentrations had the highest sensitivity corresponding to a negative temperature coefficient of about $-0.004 \mathrm{~K}^{-1}$, an absolute value that is comparable to the highest values found in metals $\left(0.0037-0.006 \mathrm{~K}^{-1}\right.$; for example the value of $0.00385 \mathrm{~K}^{-1}$ corresponds to a Pt100 sensor).

The different MWCNT concentration led to differences in sensor performance, and the sensor prepared from MWCNT/PVBC_Et 3 N dispersions containing the highest MWCNT concentration displayed a sensitivity about $1.5-2$ times lower. This result is attributed to the fact that materials fabricated with MWCNT content close to the percolation threshold are characterized by largest resistance sensitivity to temperature.[29]

$<$ Figure 9 near here $>$

An important feature of the prepared sensors is reported in figure 9, which illustrates the response of the MWCNT/PVBC_Et 3 N 1:20 sensor to a sequence of heating and cooling cycles from $20^{\circ} \mathrm{C}$ to $40{ }^{\circ} \mathrm{C}$. It appears that the resistance of the sensor well recovers to the original value after the return to the room temperature, thus indicating the good reproducibility of the sensor response. 


\section{Conclusions}

Well exfoliated MWCNT/polymer nanocomposites can be realized by using a new styrene-based ionomer, a poly(vinylbenzyl chloride) in which $78 \% \mathrm{Cl}$ atoms of the benzyl groups were replaced by $\mathrm{Et}_{3} \mathrm{~N}$. The weight percentage of CNTs in MWCNT/PVBC_Et ${ }_{3} \mathrm{~N}$ nanocomposites can be easily modulated by varying the MWCNT concentration of DMF mixtures, as demonstrated by UV-vis experiments and TGA investigations. AFM microscopy and Raman spectroscopy evidenced the realization of well exfoliated and intimate polymer mixtures containing undamaged MWCNTs. The great dispersability of MWCNTs in PVBC_ $\mathrm{Et}_{3} \mathrm{~N}$ was also reflected on the electrical resistance of nanocomposites, which showed a strong increase of conductivity for a relatively small variation of MWCNT concentration from $13.2 \mathrm{wt} \%$ to $17.6 \mathrm{wt} . \%$, thus suggesting the formation of highly percolative MWCNT/polymer networks.

The MWCNT/PVBC_Et ${ }_{3} \mathrm{~N}$ system displayed, for MWCNT contents close to the percolation threshold, a resistance sensitivity to temperature of $-0.004 \mathrm{~K}^{-1}$, an absolute value that is comparable to the highest values found in metals. It is worth to notice that the MWCNT/PVBC_Et ${ }_{3} \mathrm{~N}$ nanocomposite showed a very good reproducibility of the sensor response towards alternating heating and cooling cycles, thanks to the phase stabilization of the composite provided by the high Tg ionomer.

In conclusion, the results reported here indicated that such nanocomposite can be exploited for the realization of low-cost, small-sized and highly reproducible temperature sensors to be used in the range $20-40^{\circ} \mathrm{C}$. Future work will involve the optimization of the dispersion procedure of MWCNTs to best match the percolation threshold and thereby improve sensitivity. The possible influence of humidity on the sensor performances will be also investigated and a protecting polyimide tapes will be eventually utilized during assembly and throughout the life of the sensor. 


\section{Acknowledgements}

The financial support of European Commission (FP7 Project 317894-SWAN-iCARE) is gratefully acknowledged. Dr. Michele Alderighi is acknowledged for his help in AFM measurements.

\section{References}

[1] S. Iijima, Nature, 354 (1991) 56-58.

[2] P.M. Ajayan, O. Stephan, C. Colliex, D. Trauth, Science, 265 (1994) 1212-1214.

[3] J.M. Schnorr, T.M. Swager, Chemistry of Materials, 23 (2011) 646-657.

[4] W.A. de Heer, MRS Bulletin, 29 (2004) 281-285.

[5] M. Moniruzzaman, K.I. Winey, Macromolecules, 39 (2006) 5194-5205.

[6] N.G. Sahoo, S. Rana, J.W. Cho, L. Li, S.H. Chan, Progress in Polymer Science, 35 (2010) 837867.

[7] F. Ciardelli, S. Coiai, E. Passaglia, A. Pucci, G. Ruggeri, Polym. Int., 57 (2008) 805-836.

[8] M.T. Byrne, Y.K. Gun'ko, Advanced Materials, 22 (2010) 1672-1688.

[9] R.H. Baughman, A.A. Zakhidov, W.A. de Heer, Science, 297 (2002) 787-792.

[10] K.L. Lu, R.M. lago, Y.K. Chen, M.L.H. Green, P.J.F. Harris, S.C. Tsang, Carbon, 34 (1996) 814-816.

[11] G. Matzeu, A. Pucci, S. Savi, M. Romanelli, F. Di Francesco, Sensors and Actuators, A: Physical, 178 (2012) 94-99.

[12] N. Calisi, A. Giuliani, M. Alderighi, J.M. Schnorr, T.M. Swager, F. Di Francesco, A. Pucci, European Polymer Journal, 49 (2013) 1471-1478.

[13] L. Vaisman, H.D. Wagner, G. Marom, Advances in Colloid and Interface Science, 128-130 (2006) 37-46.

[14] R. Rastogi, R. Kaushal, S.K. Tripathi, A.L. Sharma, I. Kaur, L.M. Bharadwaj, Journal of Colloid and Interface Science, 328 (2008) 421-428.

[15] V.C. Moore, M.S. Strano, E.H. Haroz, R.H. Hauge, R.E. Smalley, J. Schmidt, Y. Talmon, Nano Letters, 3 (2003) 1379-1382.

[16] D.M. Delozier, D.M. Tigelaar, K.A. Watson, J.G. Smith Jr, D.J. Klein, P.T. Lillehei, J.W. Connell, Polymer, 46 (2005) 2506-2521.

[17] J. Wang, M. Musameh, Y. Lin, Journal of the American Chemical Society, 125 (2003) 24082409.

[18] M. Faraj, E. Elia, M. Boccia, A. Filpi, A. Pucci, F. Ciardelli, Journal of Polymer Science, Part A: Polymer Chemistry, 49 (2011) 3437-3447.

[19] M. Faraj, M. Boccia, H. Miller, F. Martini, S. Borsacchi, M. Geppi, A. Pucci, International Journal of Hydrogen Energy, 37 (2012) 14992-15002.

[20] A. Filpi, M. Boccia, A. Pucci, F. Ciardelli, e-Polymers, (2013) 001.

[21] M. Piana, M. Boccia, A. Filpi, E. Flammia, H.A. Miller, M. Orsini, F. Salusti, S. Santiccioli, F. Ciardelli, A. Pucci, Journal of Power Sources, 195 (2010) 5875-5881.

[22] G.A. Rance, D.H. Marsh, R.J. Nicholas, A.N. Khlobystov, Chem. Phys. Lett., 493 (2010) 1923.

[23] F. Wang, G. Dukovic, L.E. Brus, T.F. Heinz, Science, 308 (2005) 838-841.

[24] S. Attal, R. Thiruvengadathan, O. Regev, Analytical Chemistry, 78 (2006) 8098-8104.

[25] R. Gao, S.M. Ramirez, D.L. Inglefield, R.J. Bodnar, T.E. Long, Carbon, 54 (2013) 133-142.

[26] M.A. Pimenta, G. Dresselhaus, M.S. Dresselhaus, L.G. Cancado, A. Jorio, R. Saito, Physical Chemistry Chemical Physics, 9 (2007) 1276-1290. 
[27] Z. Morávková, M. Trchová, E. Tomsík, J. Cechvala, J. Stejskal, Polymer Degradation and Stability, 97 (2012) 1405-1414.

[28] M.S. Dresselhaus, A. Jorio, M. Hofmann, G. Dresselhaus, R. Saito, Nano Letters, 10 (2010) 751-758.

[29] S.H. Foulger, Journal of Applied Polymer Science, 72 (1999) 1573-1582. 


\section{Figure captions}

Figure 1. FT-IR spectra of PVBC and PVBC_Et ${ }_{3} \mathrm{~N}$

Figure 2. ${ }^{1} \mathrm{H}$ NMR spectrum of PVBC (a) and magnified region from 4.20 to 4.70 of ${ }^{1} \mathrm{H}$ NMR spectra of PVBC and PVBC_Et 3 N (b). The dotted curves were obtained after peak deconvolution and fitting by least-squares method $\left(\mathrm{R}^{2}=0.998\right)$.

Figure 3. Representative absorption spectra of MWCNT/PVBC_Et $\mathrm{H}_{3} \mathrm{~N}$ DMF dispersions as a function of MWNT loading and PVBC_Et 3 N DMF solution (0.4 wt.\%)

Figure 4. (a) Amount of MWNTs (wt.\%) dispersed within PVBC_Et ${ }_{3} N$; and (b) plot of absorbance value recorded at $400 \mathrm{~nm}$ as a function of MWCNTs concentration (wt.\%)

Figure 5. AFM image of MWCNT/PVBC_Et ${ }_{3} \mathrm{~N}$ nanocomposite prepared from a 1:4 by weight mixture in DMF

Figure 6. Raman spectra of MWCNT/PVBC_Et ${ }_{3} \mathrm{~N}$ prepared from 1:1 (straight line, $47.5 \mathrm{wt} . \%$ of MWCNT) and 1:4 (dashed line, 19.3 wt.\% of MWCNT) by weight mixtures in DMF $\left(\lambda_{\text {exc. }}=785\right.$ $\mathrm{nm})$

Figure 7. Logarithmic scale plot of the electrical resistance of MWCNT/PVBC_Et $t_{3} \mathrm{~N}$ as a function of MWCNT concentration

Figure 8. Percentage variation of resistance versus temperature for $\mathrm{MWCNT} / \mathrm{PVBC} \mathrm{Et}_{3} \mathrm{~N}$ nanocomposites obtained from dispersions at different MWCNT concentration

Figure 9. for MWCNT/PVBC_Et 3 N prepared from 1:20 (13.2 wt.\% of MWCNT) by weight mixture 
Schemes and figures

Figure 1

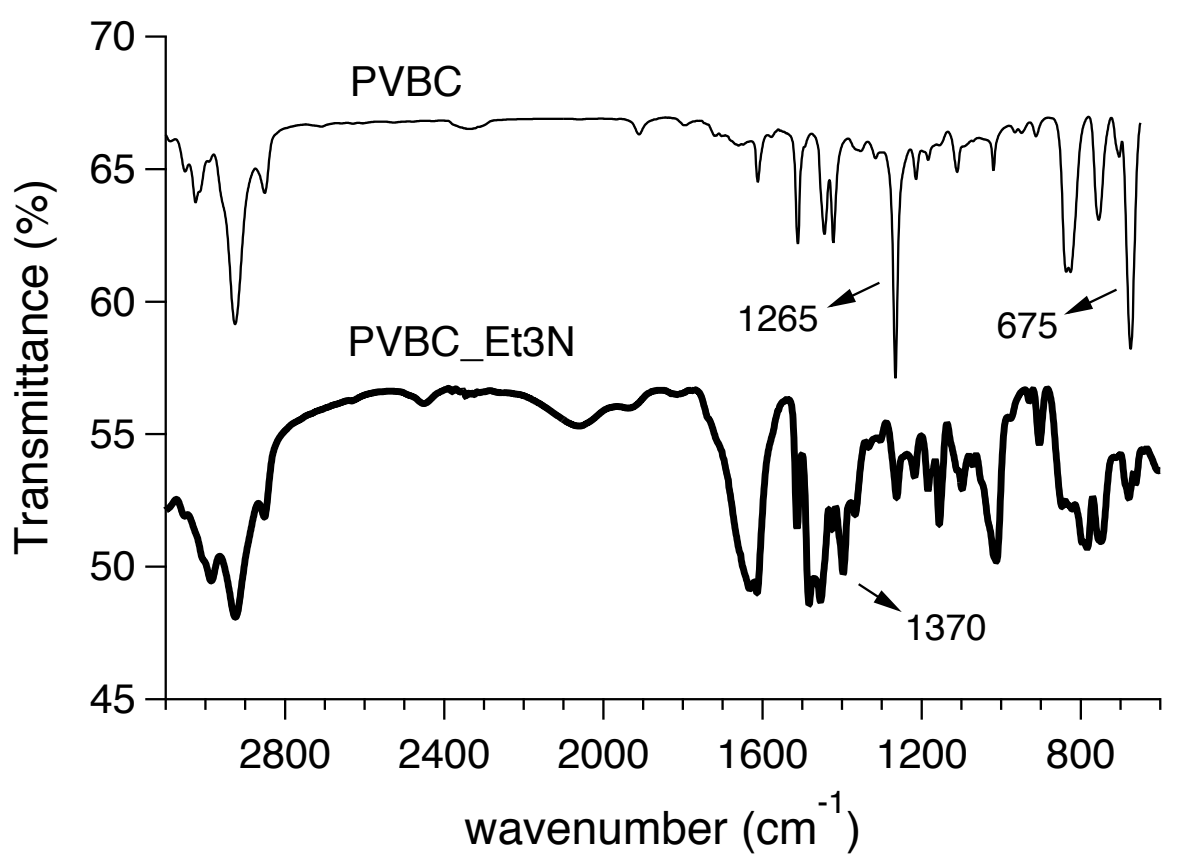


Figure 2

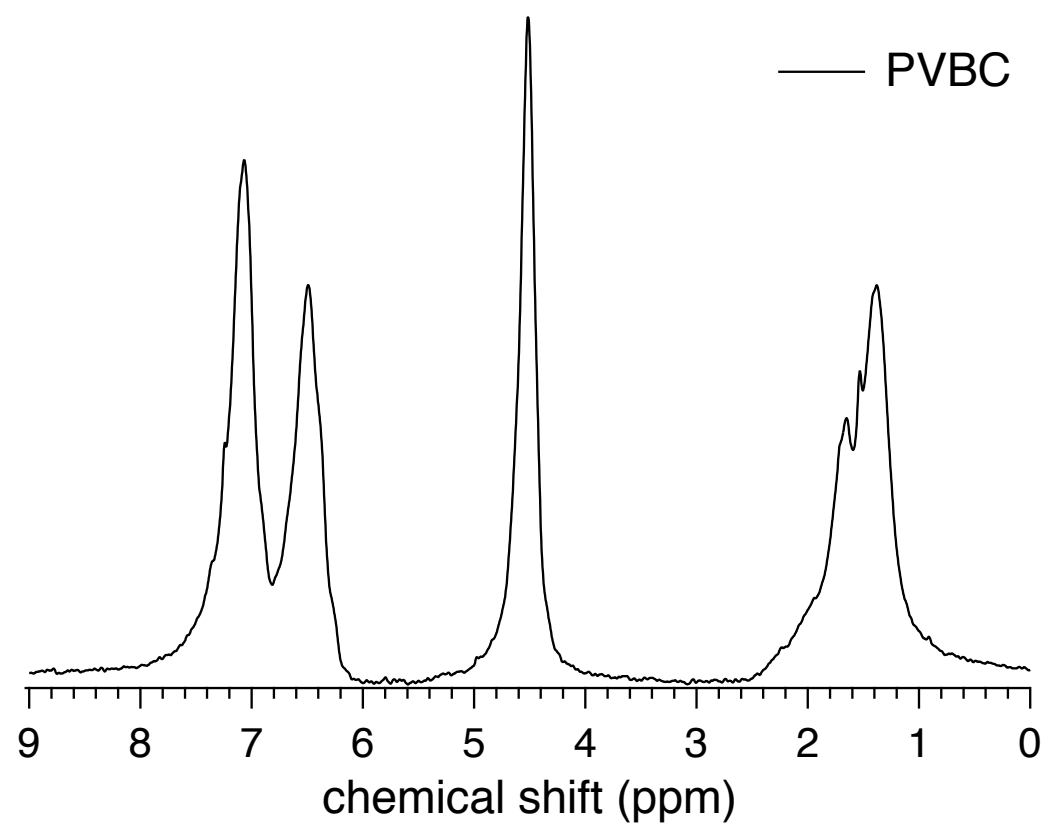

(a)

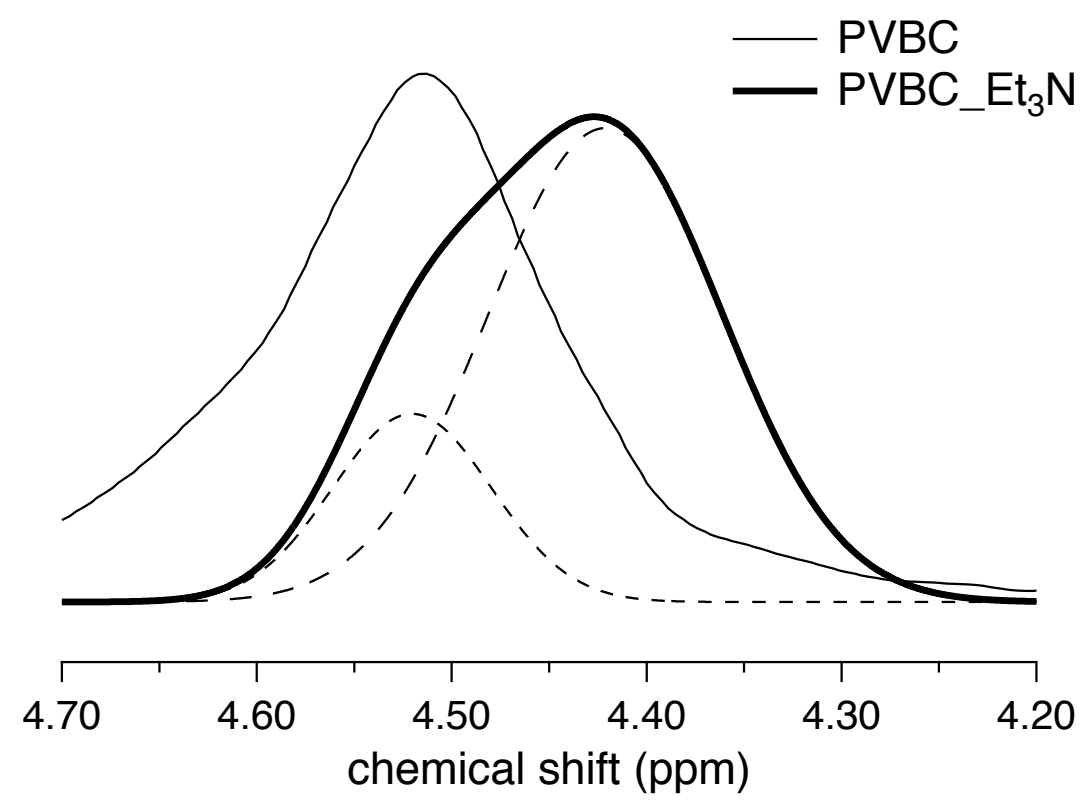

(b) 
Figure 3

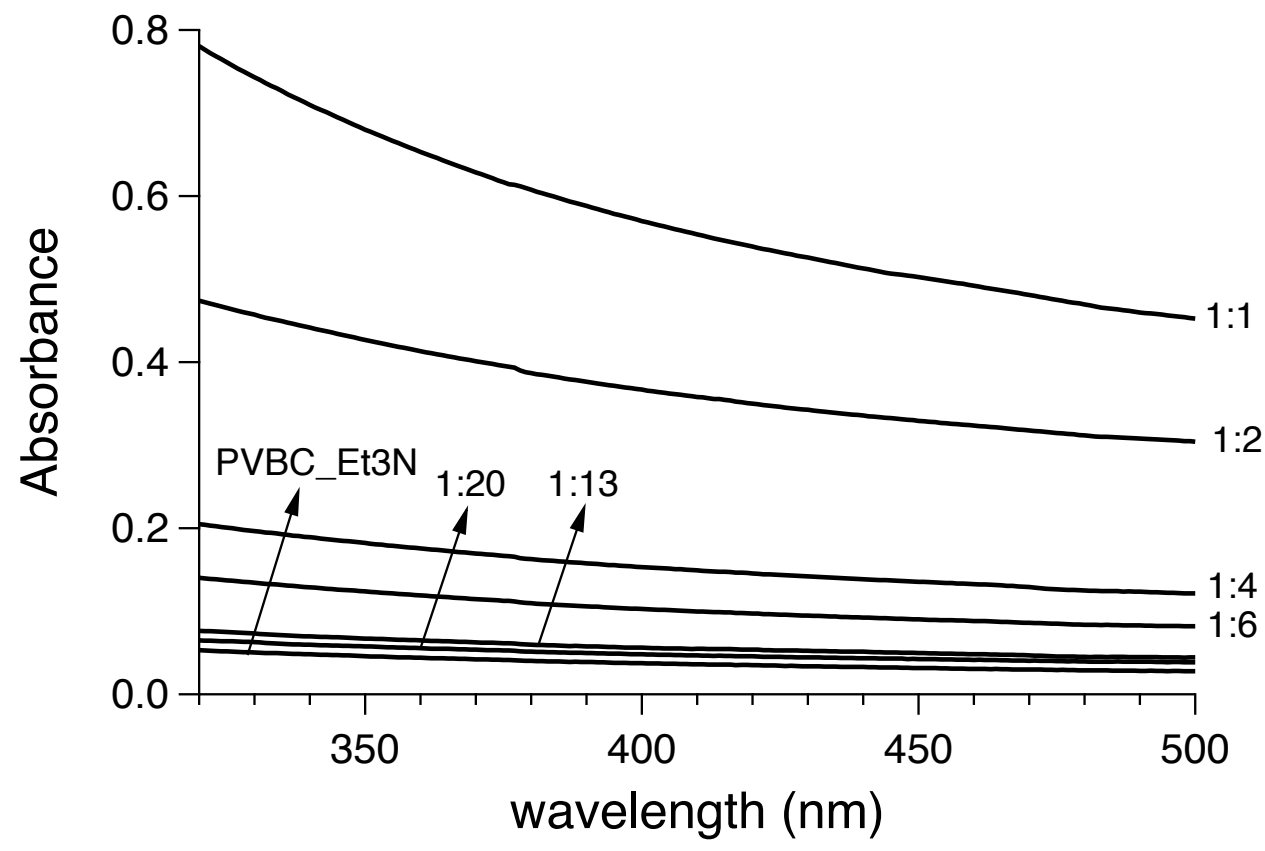


Figure 4

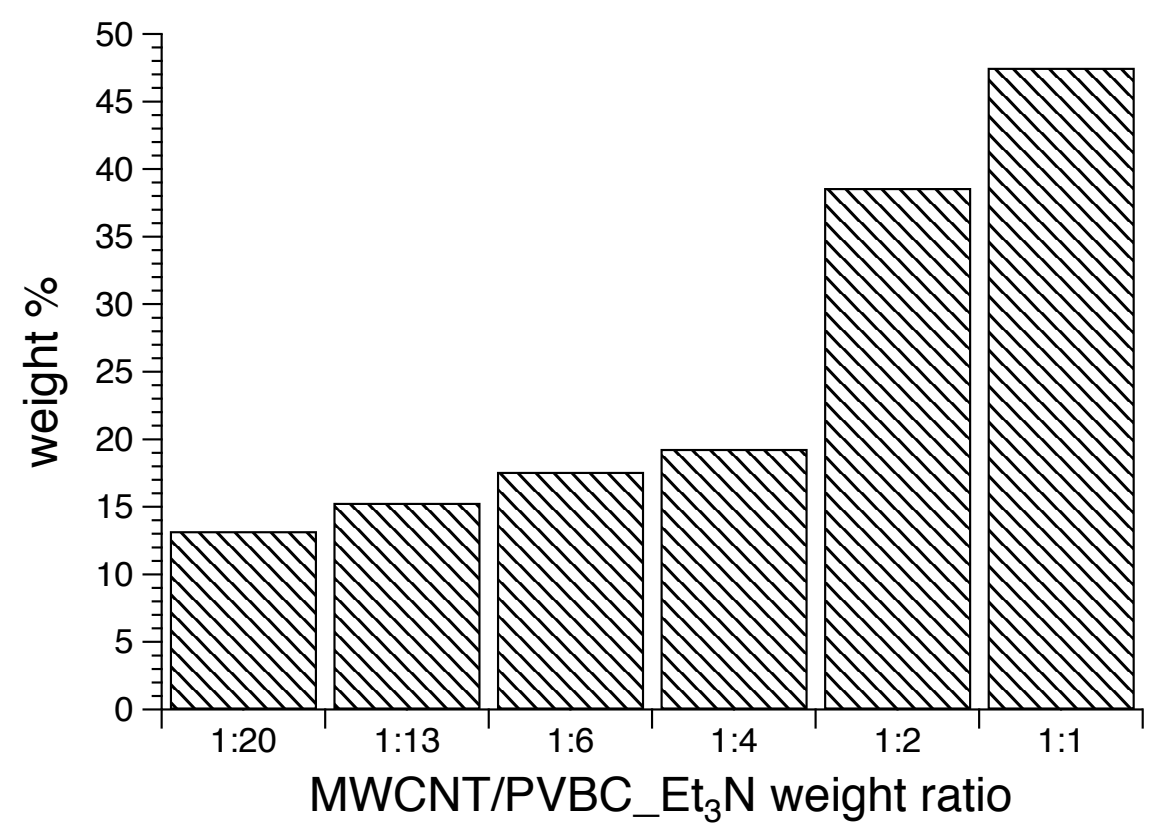

(a)

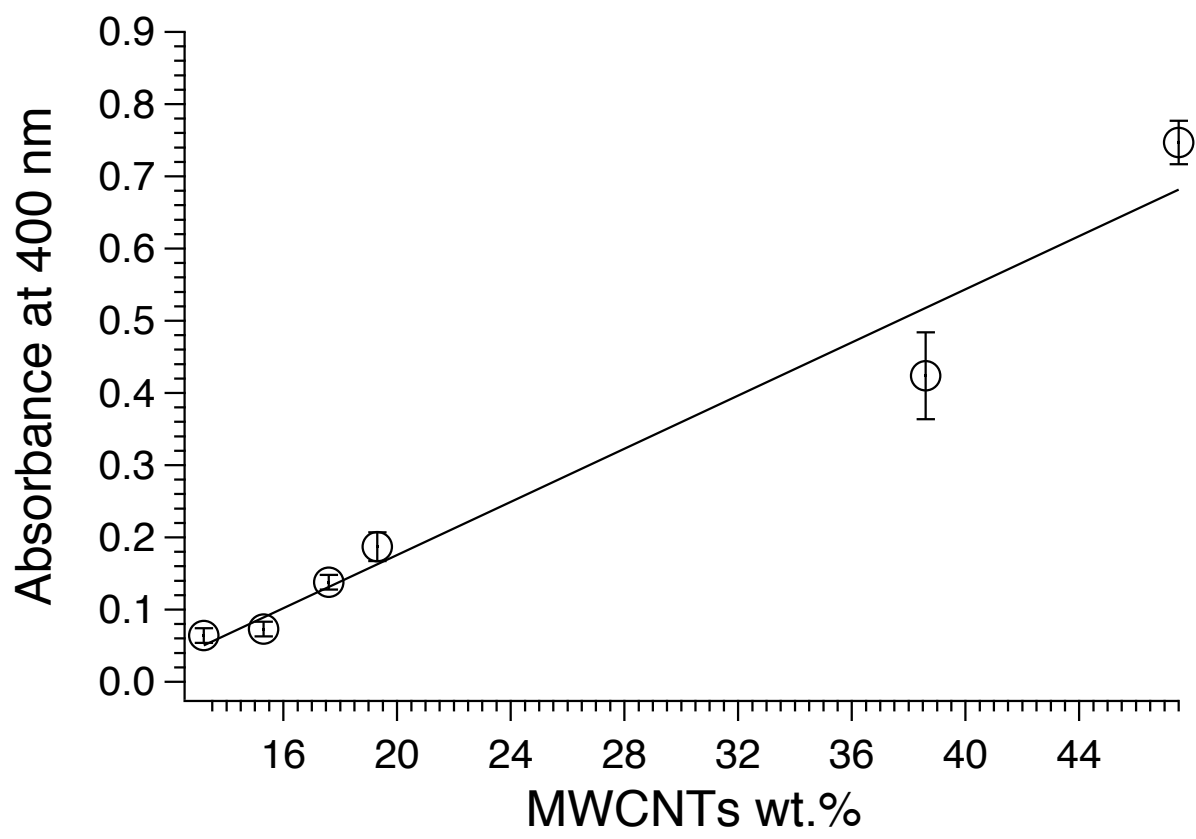

(b) 
Figure 5

Phase [R] (degree)

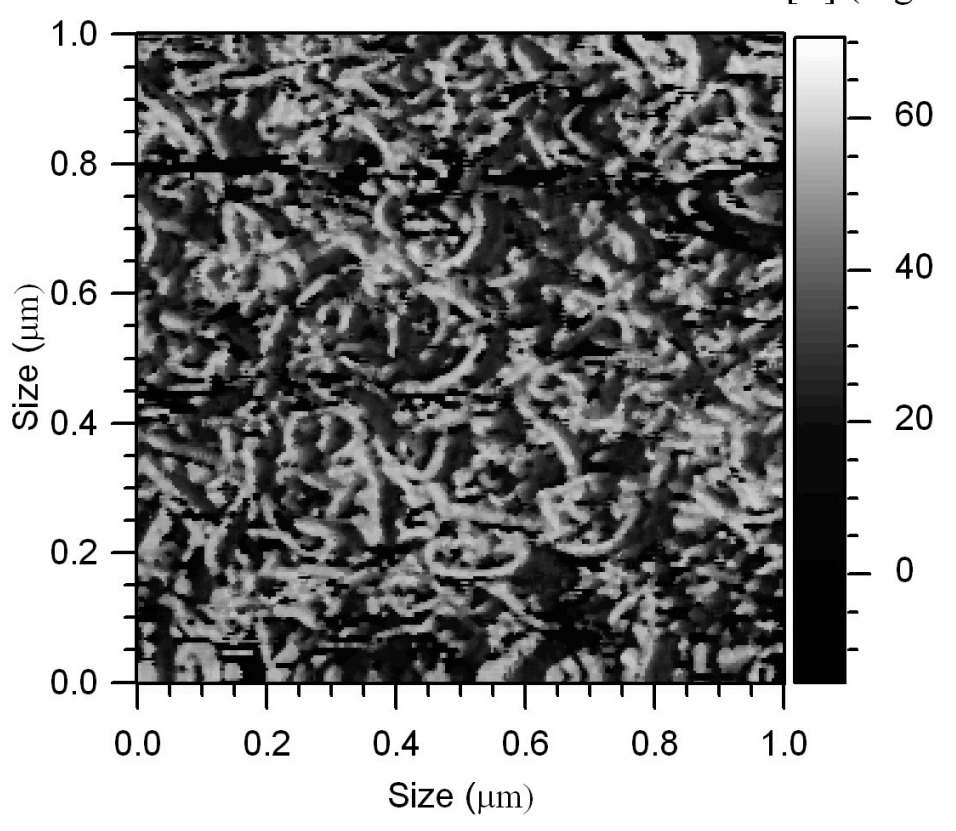

Figure 6

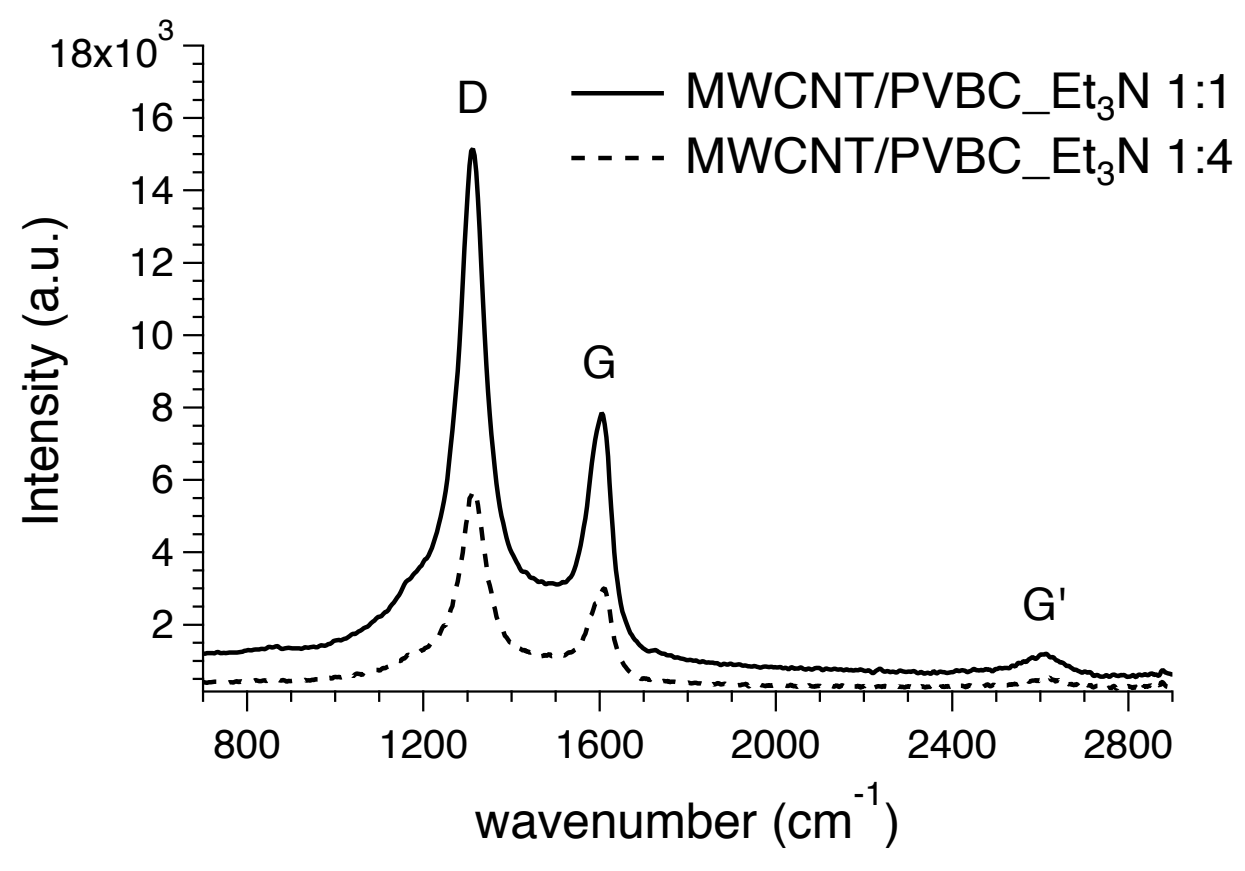


Figure 7

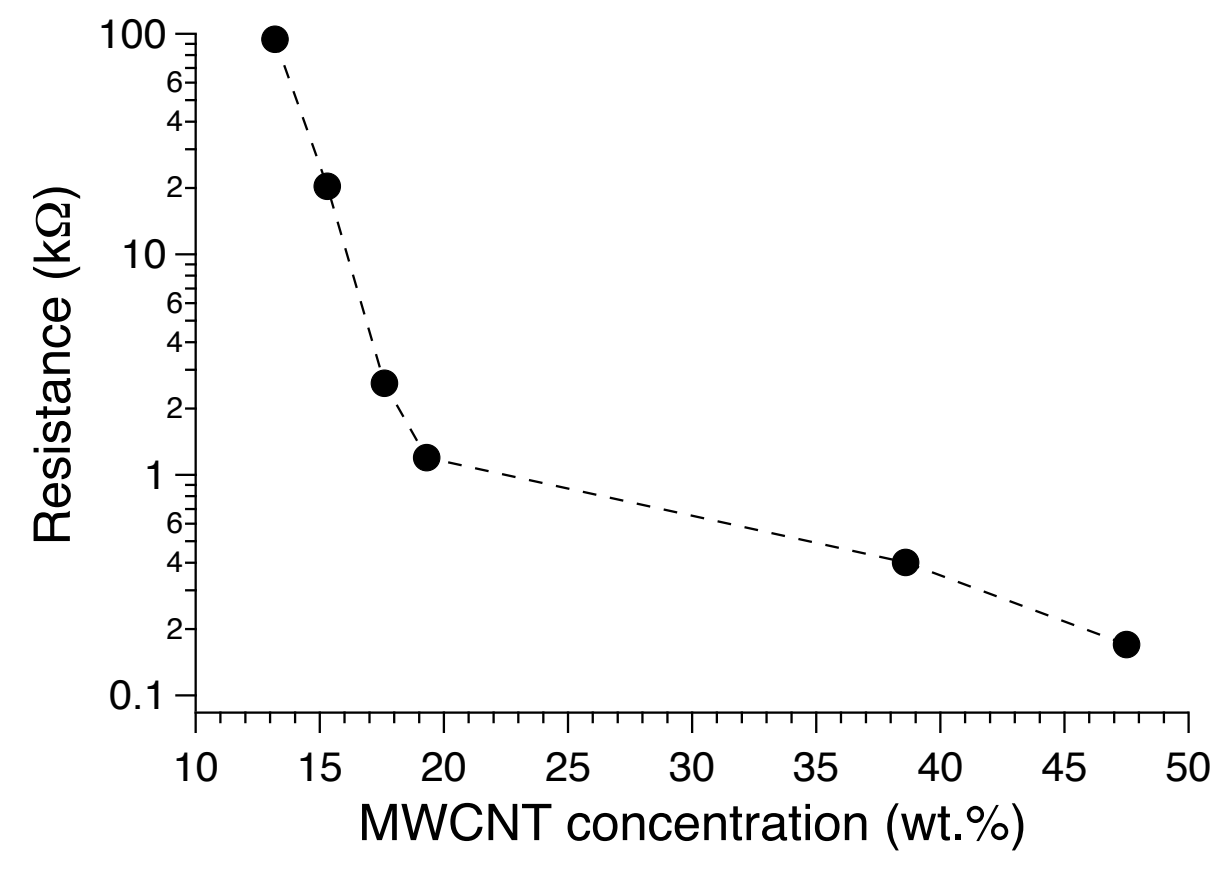

Figure 8

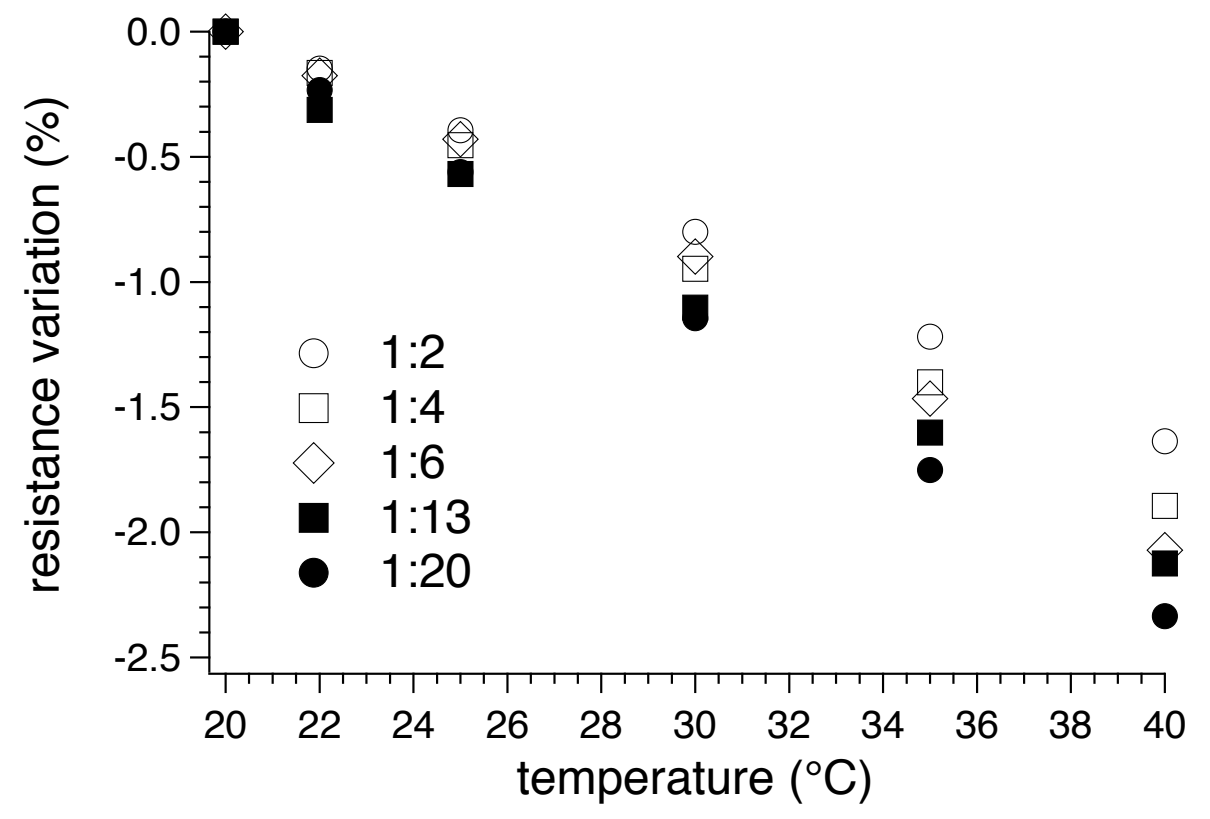


Figure 9

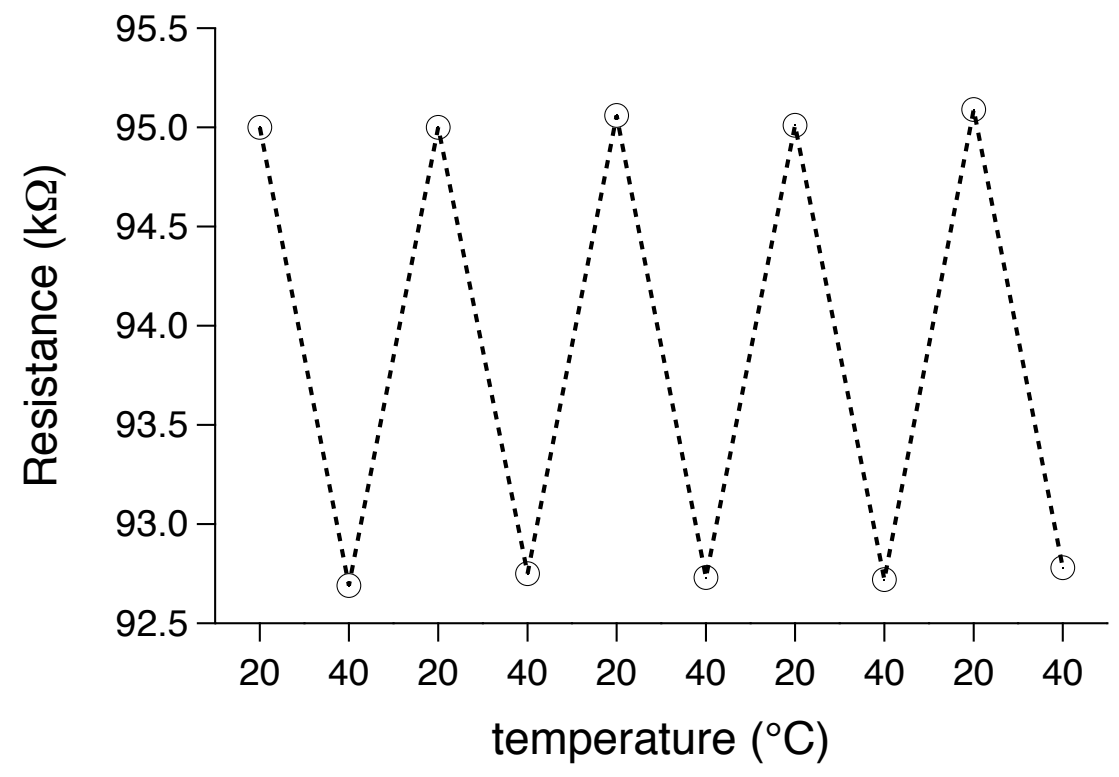




\section{Supplementary information}

\section{Alessio Giuliani, Massimo Placidi, Fabio Di Francesco, Andrea Pucci}

"A New Polystyrene-based Ionomer/MWCNT Nanocomposite for Wearable Skin Temperature Sensors"

Figure S1. Representative thermogravimetric scan of a MWCNT/PVBC_Et ${ }_{3} \mathrm{~N}$ nanocomposite with a nanotube weight percentage of about $40 \%$.

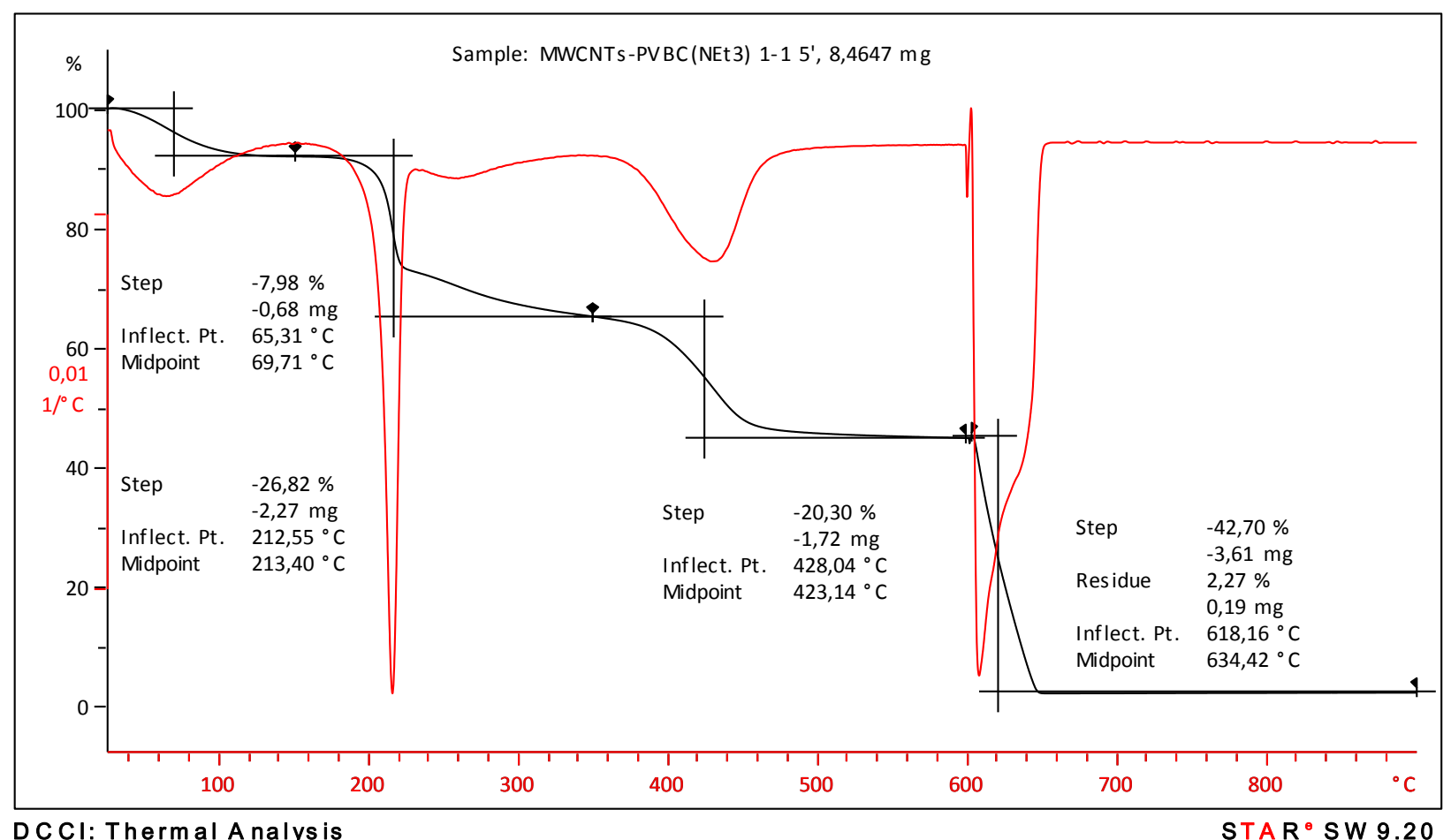


Figure S2. Schematic diagram of the sensor: (a) section and (b) top view

$$
\begin{array}{cc}
\stackrel{5 \mathrm{~mm}}{\longleftrightarrow} & 2 \mathrm{~mm} \\
\bullet & =-
\end{array}
$$

a)

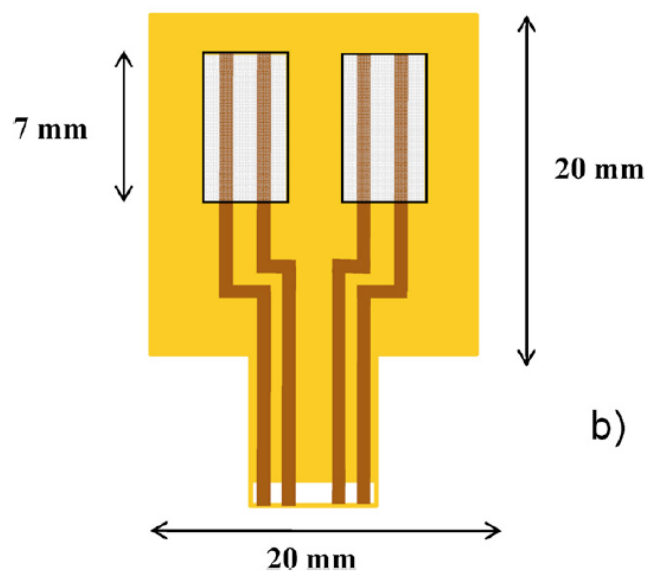

